\title{
HPR AND OPK ANGLE ELEMENT CONVERSION METHOD BASED ON AIRBORNE LIDAR ALIGNMENT AXIS ERROR CALIBRATION
}

\author{
Yexuan $\mathrm{Mu}^{1}$, Guoqing Zhou ${ }^{1}$, Xiang Zhou ${ }^{1,2, *}$, Jian Gao ${ }^{1}$, Xinyue Peng ${ }^{1}$ \\ ${ }^{1}$ Guangxi Key Laboratory of Spatial Information and Geomatics, Guilin University of Technology, No. 12 Jian’gan Road, Guilin, \\ Guangxi 541004, China \\ ${ }^{2}$ College of Mechanical and Control Engineering, Guilin University of Technology, No. 12 Jian’gan Road, Guilin, Guangxi541004, China
}

KEY WORDS: LiDAR, DGPS/IMU system, YPR, OPK, Calibration, Conversion

\begin{abstract}
:
This paper mainly uses manual calibration technology to check the elements Yaw, Pitch and Roll (YPR) in the LiDAR DGPS/IMU system and obtained the error value. Combined with the error angle, the external azimuth angle elements Kappa, Omega and Phi required by photogrammetry are obtained. The paper points out that the placement angle error will have a serious impact on the LiDAR foot position. Therefore, this paper puts forward a method to check the placement angle of the steeple roof and flat straight highway, and gives the design scheme of the optimized route to reduce the number of flights. This paper focuses on the specific process of YPR calibration, and gives a mathematical calibration model based on the influence of attitude angles Yaw, Pitch and Roll on the LiDAR foot during the flight. The placement angle error is obtained after the calibration, and the error angle matrix is used to convert the elements YPR and OPK. After checking and error correction, the point cloud obtained from adjacent airlines have achieved better coincidence effect. The experimental results show that the theory and method of YPR element calibration are correct and feasible, which simplifies the conversion process of YPR and OPK. Compared with the traditional calibration method that requires control points, this method can greatly improve the efficiency and reliability of the inspection.
\end{abstract}




\section{INTRODUCTION}

Light Detection and Ranging (LiDAR) is mainly composed of two parts, namely a LiDAR scanner that provides distance information and a pose measurement system that determines position and attitude information. The distance information obtained by LiDAR scanner combined with the attitude information obtained by IMU and the position information obtained by high precision GPS can quickly and efficiently acquire the three-dimensional information of ground targets (Zhou, 2004, 2014, 2015, 2018). At present, airborne LiDAR measurement systems are mainly used for basic measurement, urban three-dimensional reconstruction, urban environmental monitoring, coastline monitoring, forestry, urban road planning, railway and power line selection (Zhang, 2007; Zhou, 2014). As the application of airborne LiDAR technology becomes more widespread, the accuracy requirements are also increasing. Due to the integrated nature of the airborne LiDAR scanning system, the system itself will be affected by various sources of error, such as LiDAR ranging, attitude, and aircraft position. The placement angle error is usually the largest systematic error in the airborne LiDAR (Xu, 2015). The error is caused by the IMU coordinate system is not strictly parallel to the corresponding axes of the aerial photography coordinate system, resulting in the sensor attitude angle (Y,P,R) obtained by the IMU is not equal to the external azimuth angle element $(\mathrm{O}, \mathrm{P}, \mathrm{K})$ required by photogrammetry. Therefore, it is necessary to correct the placement angle error existing between the IMU coordinate system and the aerial camera coordinate system. In general, these placement angle errors are usually between 0.1 and 0.3 degrees, which is equivalent to shifting the true coordinates of the ground object by 2 to 5 meters at a relative altitude of 1000 meters (Liu, 2012; Jie, 2015). This error causes severe point cloud data migration and needs to be compensated by appropriate calibration methods.

Our research team have won a key project of innovation-driven development in Guangxi in 2018. The purpose of this project is to develop an airborne LiDAR high precision three-dimensional seabed measurement instrument (LiDAR). The schematic structure of the instrument is shown in Figure 1. My task in this project is to check the calibration axis error and convert YPR angle element into OPK angle element.

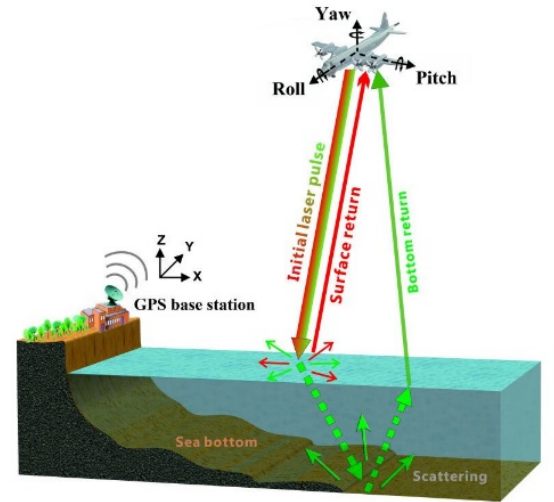

Figure 1. Dual LiDAR working diagram

In view of the above problems, this paper combines the design of route and mathematical model, and proposes a YPR calibration method without ground control points. The results of the calibration are used for the transformation of the attitude angles $\operatorname{Roll}(\Phi), \operatorname{Pitch}(\theta), \operatorname{Yaw}(\psi)$ and the outer azimuth elements $\operatorname{Omega}(\varphi)$, Phi $(\omega)$, Kappa $(K)$. This method improves the accuracy of point cloud data and simplifies the corner element conversion process.

\section{THE EFFECT OF HPR ERROR ON THE LIDAR FOOT}

Ignoring the change in the curvature of the Earth, the coordinates of the LiDAR foot in the WGS-84 coordinate system are as follows:

$$
\left[\begin{array}{l}
X_{84} \\
Y_{84} \\
Z_{84}
\end{array}\right]=R R_{\theta}\left[\begin{array}{l}
0 \\
0 \\
L
\end{array}\right]+R_{I N S}\left[\begin{array}{l}
X \\
Y \\
Z
\end{array}\right]+\left[\begin{array}{c}
X_{P} \\
Y_{P} \\
Z_{P}
\end{array}\right]
$$

Where $\left[\mathrm{X}_{84}, \mathrm{Y}_{84}, \mathrm{Z}_{84}\right]$ are the coordinates of the LiDAR foot in the WGS-84 system; $[0,0, \mathrm{~L}]$ is the position vector of the LiDAR foot in the instantaneous LiDAR beam coordinate system; $[\mathrm{x}, \mathrm{y}, \mathrm{z}]$ is the eccentric component of the LiDAR scanning mirror center to the reference point of the POS system, $\left[X_{P}, Y_{P}, Z_{P}\right]$ represents the coordinates of the POS system reference point in the WGS84 coordinate system; R represents the rotation matrix between the IMU coordinate system and the WGS84 coordinate system. It is a scanning angle rotation matrix, and each LiDAR point has a corresponding scanning angle (Xu, 2015). R is composed of RINS and RM, and RM is a placement angle rotation matrix composed of Yaw, Pitch, and Roll.

\subsection{The Effect of $\Delta$ Roll on the LiDAR Foot}

If the roll angle shift occurs during the flight of the aircraft, the LiDAR point cloud obtained by the scan will appear inconsistent with the height of the two sides as shown in Figure 2. $\Delta \mathrm{R}$ is the angular deviation of the IMU from the sensor in the direction of the flight (the $\mathrm{X}$ direction). When the flight direction is $0^{\circ}$, the route will produce a phenomenon of low left and high right. When flying in the direction of $180^{\circ}$, the direction of the high and low inclination of the route is opposite. Therefore, $\Delta \mathrm{R}$ will cause a difference in point cloud data of the same name for round-trip aerial surveys on the same route. The solid black line in the Figure indicates the true LiDAR foot point formed with the placement angle error $\Delta \mathrm{R}$, and the broken line - is the LiDAR foot point formed under ideal conditions.

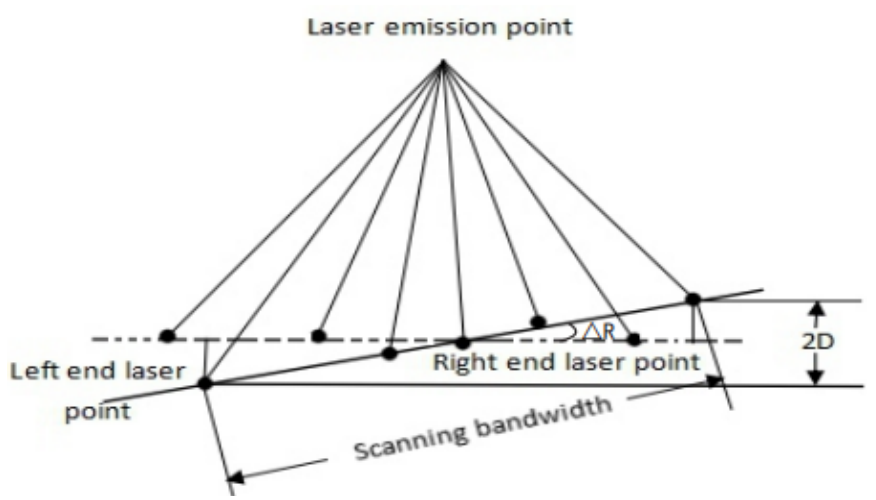

Figure 2. $\Delta$ Roll influence on the LiDAR foot point (Zhang, 2007)

\subsection{The Effect of $\triangle$ Pitch on the LiDAR Foot}

$\Delta \mathrm{P}$ is the angular deviation of the IMU from the sensor in the direction of the right wing (the Y-axis) pointing to the aircraft. The pitch angle shifts the LiDAR point cloud data forward or backward. $\Delta \mathrm{P}$ has different effects on the inclined surface and the horizontal surface. Generally, the elevation difference 
caused by the elevation angle is not seen on the horizontal ground, and the offset of the point cloud data can be seen only on the inclined surface. Therefore, the use of inclined features is more conducive to observe the effect of $\Delta \mathrm{P}$ on the LiDAR foot. As shown in Figure 3, the solid line is the LiDAR foot point obtained when there is a pitch angle error, and the broken line is the LiDAR foot point obtained when there is no pitch angle error.

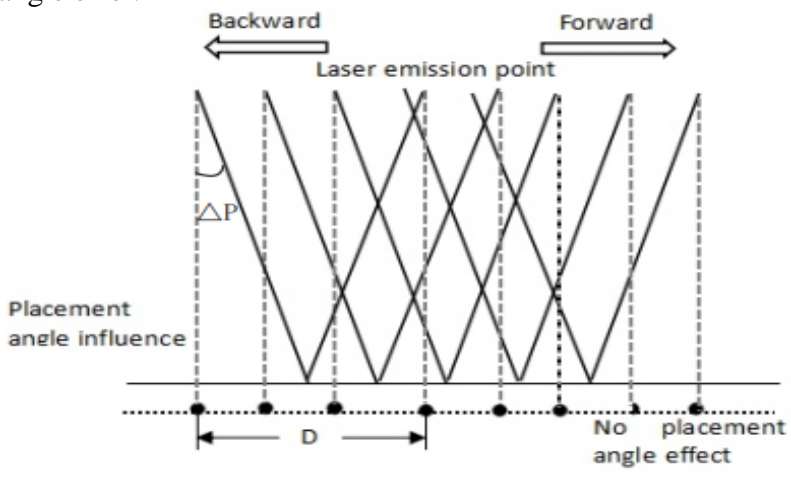

Figure 3. Effect of $\triangle$ Pitch on the LiDAR foot

(Zhang, 2007)

\section{3 $\Delta$ Yaw Influence on the LiDAR Foot}

$\Delta \mathrm{Y}$ is the yaw angle error of the IMU and the sensor in the direction of the ground ( $\mathrm{Z}$ axis), which will cause the point on the left (or right) side of the point cloud aircraft to appear in advance, and the point on the right (or left side) is appear in delay. According to the influence of $\Delta \mathrm{Y}$ on the position of the point cloud, this experiment selects the midpoint of the ridge line of the apex house as the feature point, and uses the two identical flying belts with equal overlap height to obtain the coordinates of the point along the flight direction. There is a horizontal displacement. As shown in Figure 4.

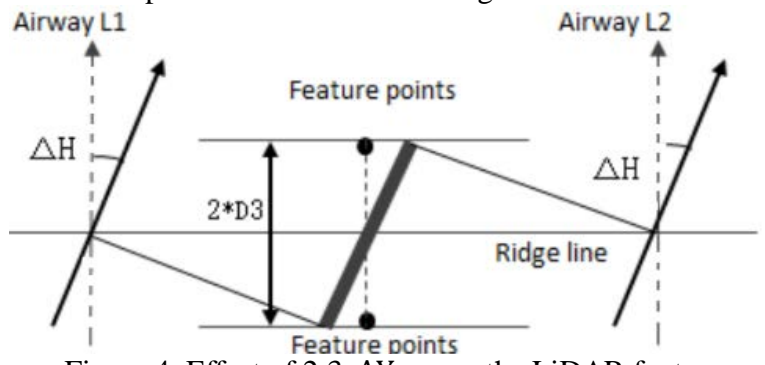

Figure 4. Effect of $2.3 \Delta$ Yaw on the LiDAR foot

\section{ANGLE CONVERSION AND CALIBRATION PRINCIPLE}

The $\operatorname{Roll}(\Phi), \operatorname{Pitch}(\theta)$, and $\operatorname{Yaw}(\psi)$ acquired by the IMU must be converted to the outer azimuth elements required for photogrammetry. Due to systematic errors, especially Placement angle errors. Therefore, the converted azimuth elements of the photo cannot be accurately oriented, and the placement angle error correction must be performed. According to the characteristics of the specific features in different flight routes, the mathematical calibration model is established by measuring the difference between the features of the same name of the overlapping navigation belts, and the placement angle is calculated by loop iteration. The results of different calibrations are different. The order of the examinations in this paper is: $\Delta$ Roll, $\Delta$ Pitch, $\Delta$ Yaw. In the loop iteration process, $\Delta$ Roll, $\Delta$ Pitch, $\Delta$ Yaw are sequentially determined, and the point cloud coordinates are recalculated by using each of the obtained calibration values, and the next calibration value is calculated by the new point cloud coordinates. The calibration is completed until all three converge to a certain threshold.

\subsection{Roll Calibration}

If there are two routes of equal height and opposite flight direction on the same trajectory, that is, $0^{\circ}$ route and $180^{\circ}$ route. The $0^{\circ}$ route has a lower left side than the right side, and the $180^{\circ}$ route has a lower right side than the left side. Therefore the formula is derived as follows:

$$
\Delta \mathrm{R}=\arctan \frac{D 1}{2 * L}
$$

As shown in Figure 5, in the formula, D1 refers to the leftmost and rightmost elevation difference of one scanning line, and $\mathrm{L}$ is the vertical distance from the edge position point to the center of the navigation belt. The general placement angle is small, so the formula (2) is approximately equal to D1/2L. Considering that the elevation of the point on the flat road changes linearly, the flat road point cloud data is generally selected to solve the roll angle. Determine the positive and negative of the calibration value according to the flight direction, and recalculate the new LiDAR point cloud by using the calibration value calculated by formula (2), and then calculate the next calibration value with the new LiDAR point cloud until the roll angle converging to a certain threshold, the calibration is completed.

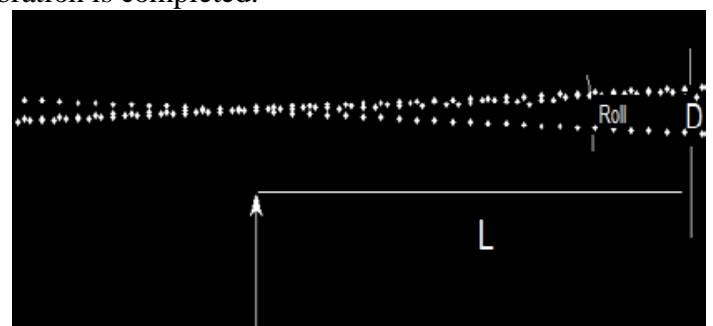

Figure 5. Schematic diagram of the Roll calibration formula

\subsection{Pitch Calibration}

Since the Pitch is an offset angle generated in the flight direction, two route data flying in the opposite direction perpendicular to the ridge line are selected for analysis. The selected feature is the apex on the track point cloud. According to Figure 6. Draw the apex roof profile and measure the distance D2 between the ridge lines, according to the formula:

$$
\Delta P=\arctan \frac{D 2}{2 h}
$$

As shown in Figure 6, D2 is the displacement of the apex of the ridge line in the horizontal direction, that is, the horizontal displacement of the point of the same name, $h$ is the average nautical height, and the correction value of the elevation angle calculated by the formula (3) $\Delta \mathrm{P}$ recalculates the coordinates of the LiDAR foot point, and then calculates the next pitch calibration value with the new point cloud coordinates, so that the calibration is performed multiple times until the elevation angle is no longer reduced, that is, when the D2 is equal to zero, the calibration is completed. 


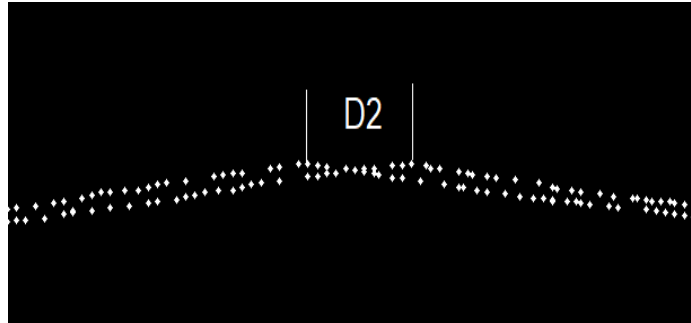

Figure 6. Pitch results in the displacement of the same name point

\subsection{Yaw Checkout}

The heading angle error will change the position of the center of the scanned object and cause the object to be deformed. The impact is greatest at the edge of the line, and no displacement occurs at the zero point of the scan. Using the two belts with equal row heights and perpendicular to the apex chamber and $30 \%$ overlap in the same direction, the same name is measured. The mathematical derivation formula is as follows:

$$
\Delta \mathrm{R}=\arctan \frac{2 * D 3}{L}
$$

As shown in Figure 7, in the formula, 2*D3 is the offset between the feature points of the same name on both sides, and the distance between the centerlines of the two flight belts. According to the previous formula $\tan \Delta \mathrm{Y}$ approximation as $\Delta \mathrm{Y}$, a plurality of heading angles are obtained, and their mean values are used as calibration values, and positive and negative are determined according to the flight direction. The LiDAR point cloud data is recalculated using the calculated value of Yaw and multiple calibrations are performed until the two apex houses overlap.

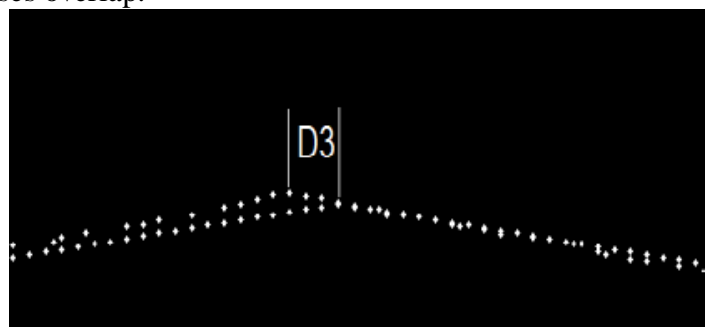

Figure 7. Yaw results in a displacement of the same name

The placement angle error obtained by the calibration will be used for the transformation of the IMU attitude angles $\operatorname{Roll}(\Phi)$, Pitch $(\theta)$, Yaw $(\psi)$ and the external azimuth elements Omega $(\varphi)$, Phi $(\omega)$, Kappa $(K)$, so The resulting conversion model is as follows:

$$
\mathrm{R}(\varphi \omega \mathrm{K})=R(\Phi, \theta, \psi) R(\Delta R, \Delta P, \Delta H)
$$

In the above formula, the model simplifies the conversion process between YPR and OPK.

\section{EXPERIMENT AND RESULT ANALYSIS}

\subsection{Route Design}

The experimental area was selected in the area of multi-peaked houses and straight road. According to the analysis of the influence of the placement angle on the LiDAR foot point and the placement angle characteristics, the route is designed as follows:

a) Passing from above the road and perpendicular to the direction of the road, designing two routes with opposite directions of heading overlap, $\mathrm{AB}$ and $\mathrm{CD}$;

b) Two overlapping routes passing through the top of the apex, opposite directions, and required to be perpendicular to the direction of the ridge of the apex;

c) Two parallel routes passing through the top of the apex, in the same direction, and required to be perpendicular to the direction of the ridge of the apex (30\% overlap of adjacent routes); the heading angle is generally difficult to check, because The calibration baseline (the distance between the center point of the feature and the bottom of the flight) is generally short, resulting in a small amount of movement of the object, which results in a large error in the correction of the heading angle. Here, the route is designed on both sides of the object, so the distance between the center points of the features obtained by the two flights is twice that of a single flight, and the heading angle can be accurately calculated.

This experiment considers the reduction of the number of flights and the improvement of work efficiency, and selects the parallel of the road and the ridgeline. Therefore, the above design is made, a) and b) can share the route $\mathrm{AB}$ and the route $\mathrm{CD}$, and $\mathrm{b}$ ) and c) can share the route CD. The route design diagram is shown in Figure 8.

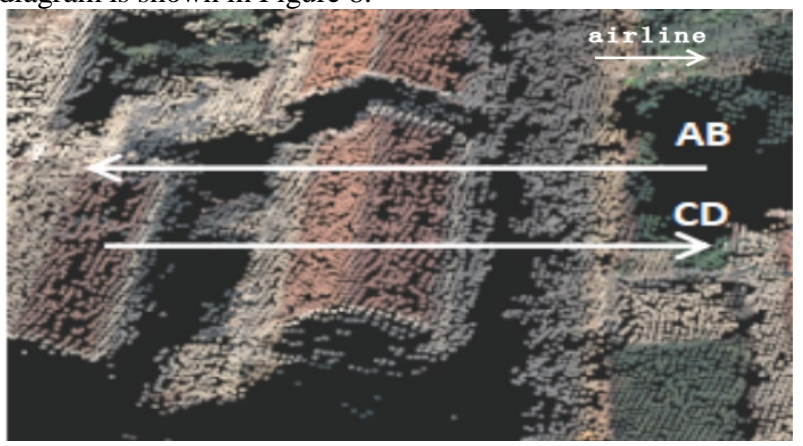

Figure 8. Flight airline design

\subsection{Results and Analysis}

The experimental data in this paper comes from the southern part of Guangxi Province - Beihai Weizhou Island with a total area of about $41 \mathrm{~km}^{2}$. The instrument is a dual-frequency airborne LiDAR detector, and the P-750 XSTOL of the Anshan Eagle Navigation Co., Ltd. of Liaoning Province is selected for the flight platform.

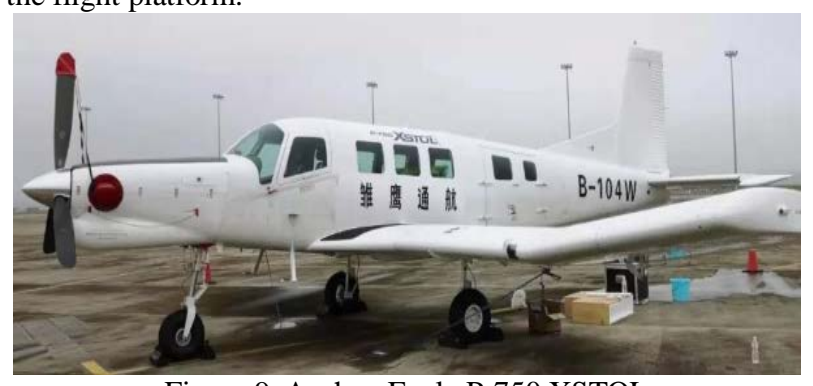

Figure 9. Anshan Eagle P-750 XSTOL

Finally, the point cloud coordinates are corrected by using the obtained placement angle parameters, and the error comparison in the point cloud before and after the calibration is shown in Table 1. After the inspection, the placement angle is used to correct the point cloud coordinates, and the cusp room after the inspection is compared with that before the inspection. From Figure 10, the degree of coincidence of the point cloud before and after the inspection can be found. 
Table 1. Comparison of point cloud accuracy

\begin{tabular}{llll}
\hline mean error & abscissa & ordinate & elevation \\
\hline before & 0.0451 & 0.0711 & 0.2045 \\
after & 0.0340 & 0.0321 & 0.0956 \\
\hline
\end{tabular}
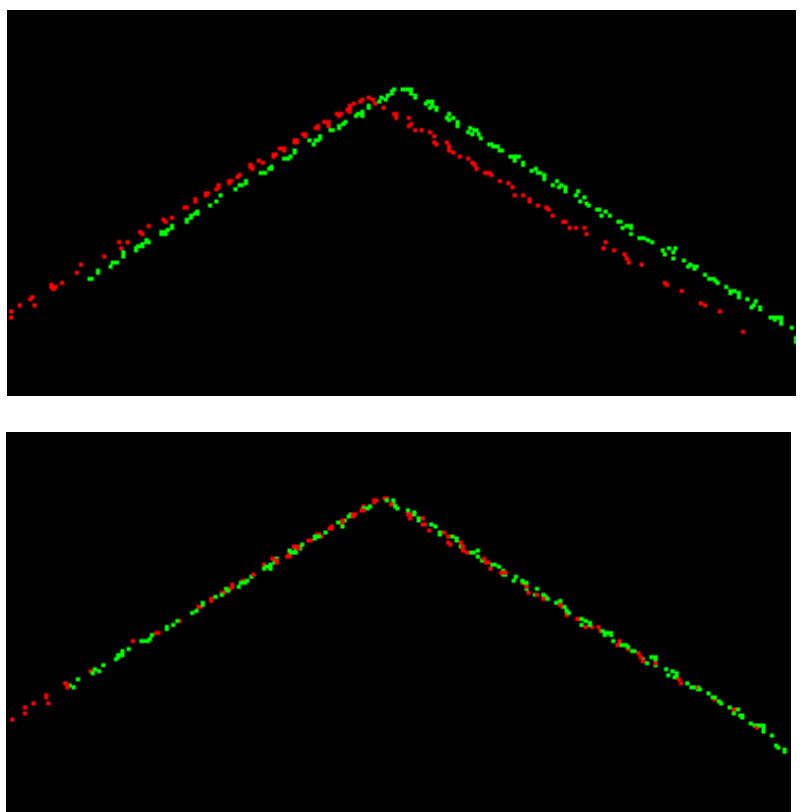

Figure 10. Front and rear cusp point clouds before and after IMU calibration

After calibration, $\Delta \mathrm{R}=0.34^{\circ}, \Delta \mathrm{P}=0.42^{\circ}, \Delta \mathrm{Y}=0.09^{\circ}$, it is used to convert $\operatorname{Omega}(\varphi), \operatorname{Phi}(\omega), \operatorname{Kappa}(\mathrm{K})$ corresponding to corner elements $\operatorname{Roll}(\Phi)$, $\operatorname{Pitch}(\theta)$, and Yaw $(\psi)$, The OPK value after RPY conversion is shown in Figure 11.

\begin{tabular}{|c|c|c|c|c|c|c|}
\hline \multicolumn{7}{|c|}{$\Delta R=0.3424^{\circ}, \Delta P=0.4216^{\circ}, \Delta Y=0.0859^{\circ}$} \\
\hline point & $\operatorname{roll}\left[{ }^{\circ}\right]$ & $\operatorname{pitch}\left[{ }^{\circ}\right]$ & $\operatorname{Yaw}\left[{ }^{\circ}\right]$ & $\varphi\left[^{\circ}\right]$ & $\omega\left[{ }^{\circ}\right]$ & $\kappa\left[{ }^{\circ}\right]$ \\
\hline 001 & -1.32 & -0.50 & -26.44 & -1.59 & 0.46 & 3.09 \\
\hline 002 & -1.35 & -0.46 & -27.53 & -1.58 & 0.48 & 3.12 \\
\hline 003 & -1.33 & -0.41 & -26.96 & -1.57 & 0.47 & 3.07 \\
\hline 004 & -1.34 & -0.39 & -26.87 & -1.58 & 0.48 & 3.12 \\
\hline & & & & & & \\
\hline 320 & -1.37 & -0.61 & -26.43 & -1.56 & 0.46 & 3.11 \\
\hline 321 & -1.45 & -0.57 & -26.47 & -1.59 & 0.46 & 3.07 \\
\hline 322 & -1.35 & -0.45 & -26.56 & -1.52 & 0.47 & 3.08 \\
\hline 323 & -1.39 & -0.44 & -26.55 & -1.54 & 0.46 & 3.10 \\
\hline
\end{tabular}

Figure 11. OPK obtained after YPR conversion

\section{CONCLUSION}

The airborne POS system adopts inertial navigation and GPS positioning technology to directly determine the external orientation elements of aerial camera, which can greatly reduce the workload of aerial remote sensing field control joint survey and shorten the period of aerial survey mapping (Yuan, 2006). The airborne LiDAR IMU is not parallel to the corresponding axis of the LiDAR scanning system, resulting in a placement angle error between the two axes. Considering the effect of Placement angle on the LiDAR point cloud, it is necessary to check the angle element YPR before the airborne LiDAR system works. The results are used to convert angle YPR to angle OPK. This experiment verifies the feasibility of the proposed method, which has an absolute advantage over Optech's use of the fork route method. This method does not require ground control points, and has achieved good results for the inspection of Roll, Pitch and Yaw of special features. This method can be used for airborne LiDAR calibration flights under unfair flight conditions. As long as there is a straight road and a number of spires on the side of the road, you can complete the basic system inspection tasks. With the development of airborne LiDAR technology, a simpler and more efficient method needs to be studied. The inspection area of this experimental constituency is more specialized, so the control point is still needed in the case of high accuracy requirements.

\section{ACKNOWLEDGEMENT}

This paper is financially supported by the National Natural Science of China under Grant numbers 41431179,41961065; Guangxi Innovative Development Grand Grant under the grant numbers GuikeAA18118038, GuikeAA18242048; the National Key Research and Development Program of China under Grant number 2016YFB0502501 and the BaGuiScholars program of of Guangxi (Guoqing Zhou).

\section{REFERENCES}

Guoqing Zhou, Xiang Zhou, Lieping Zhang, Biao Zhang, Chun tao Yang, Yilong Liu, Mingyan Li,2015:Development of Time I nterval Measuring System with MultiChannel for Array LiDAR , Chinese Journal of Electron Devices 38(1), 166-173.

Jie, C., Chunlei, X., \& Jing, L. I., 2015: Calibration of airborne lidar cloud point data with no calibration field. Remote Sensing for Land \& Resources 27(4), 27-33.

Liu JJ, Huang HP, Miao Z X, et al., 2012: Discussion on airborne LiDAR system boresight self-calibration. Surveying and Mapping 35 (2), 74-77.

Xu, P., Kun, S., \& Zhengxiang, Z, 2015: Error analysis and calibration methods of boresight misalignment in airborne liDAR measurement system. Geotechnical Investigation \& Surveying.

Yuan Xiuxiao, Yang Fen, Zhao Qing. et al., 2006: Bore sight Misalignment Calibrition of Integrated DGPS/IMU System. Geomatics and Information Science of Wuhan University 3l (12), 10391043

Zhou, G., Song, C., Simmers, J, et al., 2004: Urban 3D GIS From LiDAR and digital aerial images. Computers\& Geosciences 30 (4),345-353.

Zhou, G., \& Zhou, X, 2014: Seamless fusion of LiDAR and aerial imagery for building extraction. IEEE Transactions on Geoscience and Remote Sensing 52(11), 7393-7407.

Zhou,G., \& Zhou,X, 2018: Technology and Applications for Array LiDAR Imager (Chinese). Wuhan Univ. Press, 978-7-307-19683-4.

Zhang Xiaohong, 2007: Air-borne technology theory and methods. Wuhan University Press, Wuhan.

Zhou, G., Bo, Y., Zhang, W., Tao, X., Wei, Z., \& Tao, Y., et al., 2014: Simulation study of new generation of airborne scannerless LiDAR system. Geoscience \& Remote Sensing Symposium,524-527. 